\title{
The advantage of inactivated vaccine for preventing the Omi- cron outbreak
}

\author{
X. Lian ${ }^{1}$, J. Huang ${ }^{1 *}$, L. Zhang ${ }^{1}$, Y. Zhao ${ }^{1}$, D. Wang ${ }^{1}$, S. Gao', R. Wang ${ }^{1}$, X. Song ${ }^{1}$ \\ 1 Collaborative Innovation Centre for West Ecological Safety (CIWES), Lanzhou University, Lanzhou, 730000, \\ China; \\ * Correspondence: Jianping Huang, Collaborative Innovation Center for Western Ecological Safety, College of \\ Atmosphere Sciences, Lanzhou University, Lanzhou, 730000, China. email: hjp@lzu.edu.cn
}

\begin{abstract}
The COVID-19 pandemic has now become very severe as never before due to the overwhelming spread of Omicron. We found that Omicron outbreak can be effectively prevented by inactivated vaccines, which averted an outbreak of more than 1.6 million people in Hangzhou, China. The 36 mutations in the target spike protein of Omicron neutralizing antibody enable it to evade the immune protection afforded by vaccines. This is because that mRNA and adenovirusvector vaccines are designed to recognize the spike (S) glycoprotein of the SARS-CoV-2 wild-type (WT) strain. However, Inactivated vaccines contain the whole viral antigens and remain stable in their recognition of newly emerging variants of SARS-CoV-2. Our study confirmed the advantage of inactivated vaccines in the face of highly mutated Omicron variant and provided a basis for the development of effective vaccines to prevent future long-term transmission, mutation and recurrence of SARS-CoV-2.
\end{abstract}

Keywords: COVID-19; Omicron; inactivated vaccines

\section{Introduction}

South Africa issued an Omicron (B.1.1.529) mutant alert on November 24, 2021 [1]. This variant is the most infectious of the SARS-CoV-2 variants so far [2], which quickly spread around the world a few weeks later and is breaking the COVID-19 infections records in Europe, North America, Africa and Australia. Most countries have tightened border controls to slow the spread of Omicron. However, the policy of travel restriction among a few countries has not shut Omicron out entirely. Due to numerous mutations in the spike protein, Omicron may significantly limit the antibody-mediated neutralization, potentially posing a threat to the effectiveness of current vaccines and therapeutic antibodies[3].

At present, vaccines validated by the World Health Organization (WHO) mainly include inactivated whole-virion vaccines (CoronaVac, BBIBP-CorV, and Covaxin), mRNA vaccines (BNT162b2 and mRNA-1273), and adenovirus-vector vaccines (Ad26.COV2.S, AZD1222, Covishield) [4]. More than 8.8 billion vaccine doses have been administered worldwide, raising hope that the pandemic can be brought under control [5]. However, Omicron variants again complicate the situation. The decline of the serum antibody levels is more rapid in the vaccinated individuals compared to naturally infected patients [6]. Meanwhile, marked differences exist among approved vaccine regimens $[7,8]$. In this study, we compared vaccine protection and epidemic trends in China and other countries. Furthermore, we analyzed the impact of various vaccines on the epidemic development and explained the transmission differences of Omicron in countries. Our results provide new insights into vaccine evaluation and long-term preparation for future emergence of highly mutated viruses.

\section{Materials and Methods}




\subsection{Data source}

We selected the data of vaccines approved for use in each country as the theoretical basis for the global distribution of inactivated vaccines, as there are no comprehensive data on global vaccination rates for each type of vaccine. The COVID-19 dataset is collected from the COVID-19 Data Repository by the Center for Systems Science and Engineering (CSSE) at Johns Hopkins University which illustrates the number of confirmed COVID-19 cases, deaths, and recovered cases for all affected countries in real-time (https://github.com/CSSEGISandData/COVID-19). The number and type of vaccines approved for use in each country are derived from: https://covid19.trackvaccines.org/trialsvaccines-by-country/. As of 10 February, 2022, 146 countries have shared 1,116,011 Omicron gene sequences from sample collection for public access via GISAID EpiCoV. Gene sequence data for each country were obtained from https://www.gisaid.org/hcov19-variants/.

\subsection{Test of abrupt change}

We used the moving t-test technique to identify the abrupt change point of the global pandemic to further determine the impact of Omicron variant on the global pandemic. The moving t-test is a statistical method for detecting abrupt change by examining whether differences in sample mean values are significant. It is assumed that the two subsequences in a certain time series obey the same normal population variance, and if their mean value differences exceed a certain level of significance, the two mean values can be considered to have an abrupt change. The length of the window in this study is 14 days. Data used is updated to 19 January 2022. An abrupt change is considered when the t-test value is greater than 5 . If the interval between the abrupt change point and the latest update date is less than the window length, then the absolute value is chosen as the window length. The $\mathrm{p}$-values and $\mathrm{t}$-values can be obtained by applying the student $\mathrm{t}$-test to the right and left windows.

\subsection{The modified SEIR model}

In this study, the second version of the Global Prediction System of COVID-19 Pandemic (GPCP) developed by Lanzhou University (http://covid-19.lzu.edu.cn/) was used to analyze the impact mechanism of Omicron through parameter setting in combination with globally reported case data [9]. The system uses a modified version of the susceptible, exposed, infectious, recovered (SEIR) epidemiological model, whose parameters are generated from the inversion of actual epidemic data. The theoretical framework of modified SEIR model is based on the division of the human host population into categories containing susceptible cases $(S)$, protected cases $(P)$, potentially infected cases $(E)$, infected cases $(\mathrm{I})$, quarantined cases $(\mathrm{Q})$, recovered cases $(R)$, and cases of mortality (D). The sum of the seven groups is equal to the total population $(\mathrm{N})$. The dynamics of each group are determined by the parameters protection rate () , infection rate $(\beta)$, average latent time $(\gamma)$, average quarantine time $(\delta)$, cure rate $(\lambda)$, and mortality rate $(\kappa)$. The epidemiological model is based on the model of Peng et al. which contains the following equations [10]:

$$
\begin{gathered}
\frac{d S(t)}{d t}=-\frac{\beta(t) I(t) S(t)}{N}-\alpha S(t) \\
\frac{d P(t)}{d t}=\alpha S(t) \\
\frac{d E(t)}{d t}=\frac{\beta(t) I(t) S(t)}{N}-\gamma E(t) \\
\frac{d I(t)}{d t}=\gamma E(t)-\delta I(t)
\end{gathered}
$$




$$
\begin{gathered}
\frac{d Q(t)}{d t}=\delta I(t)-\lambda(t) Q(t)-\kappa(t) Q(t) \\
\frac{d R(t)}{d t}=\lambda(t) Q(t) \\
\frac{d D(t)}{d t}=\kappa(t) Q(t)
\end{gathered}
$$

As for the epidemic in Hangzhou city, we simulated the development trend of the epidemic under different government control measures by modifying the protection days. According to the literature, the Omicron variant is 2.8 times as contagious as the delta variant, and the transmission of the Omicron variant strain is simulated by setting the value of $\beta$ [11].

\section{Results}

\subsection{Epidemic trend in countries}

The comparison of epidemic trend in non-inactivated vaccination and inactivated vaccination countries is shown in Figure 1. The significant abrupt change happened in countries that had not vaccinated inactivated vaccines. The average number of new cases within 20 days is about 24,000 , more than 3 times the number before the abrupt change. However, countries using inactivated vaccines have seen a flattening epidemic trend and the average number of new cases within 20 days is only about 3,400, an increase of only about 2,000 compared to pre-abrupt change levels (about 2/17 of those in non-inactivated vaccination countries). Notably, inactivated vaccines are not available in the top 10 countries in the world with Omicron genome sequences (Table 1).

An important reason for the rapid global spread of Omicron VOC (variants of concern) is immune evasion, which may have an $88 \%$ chance of escaping the current vaccines, while the broad spectrum of inactivated vaccines could effectively contain Omicron outbreaks in some countries [11]. The Omicron variant has 36 mutations in the target spike protein of the neutralizing antibody that may influence the effectiveness of existing noninactivated vaccines, as all of them are designed to recognize the spike (S) glycoprotein of the SARS-CoV-2 wild-type (WT) strain [12]. Omicron has been shown to escape antibody neutralization of the BNT162b2 messenger RNA vaccine [13]. The monoclonal antibodies (mAbs) from Eli Lilly, AstraZeneca, Regeneron mAb cocktail, Celltrion, and Rockefeller University may also be seriously compromised by Omicron variant11. In addition, inactivated vaccines contain additional viral antigens, which may also elicit T-cell immunity, resulting in higher responses $[14,15]$. Studies have shown that CoronaVac induces higher $\mathrm{CD} 4^{+}$and $\mathrm{CD} 8^{+} \mathrm{T}$-cell responses to the structural protein than BNT162b2 [14]. Novel Coronavirus epitopes recognized by human T-cells are not limited to spike proteins or receptor binding domains (RBD) of spike proteins $[16,17]$. Therefore, most T-cells remain stable in their recognition of newly emerging variants of SARS-CoV-2, further confirming the advantage of inactivated vaccines against highly mutated virus. 


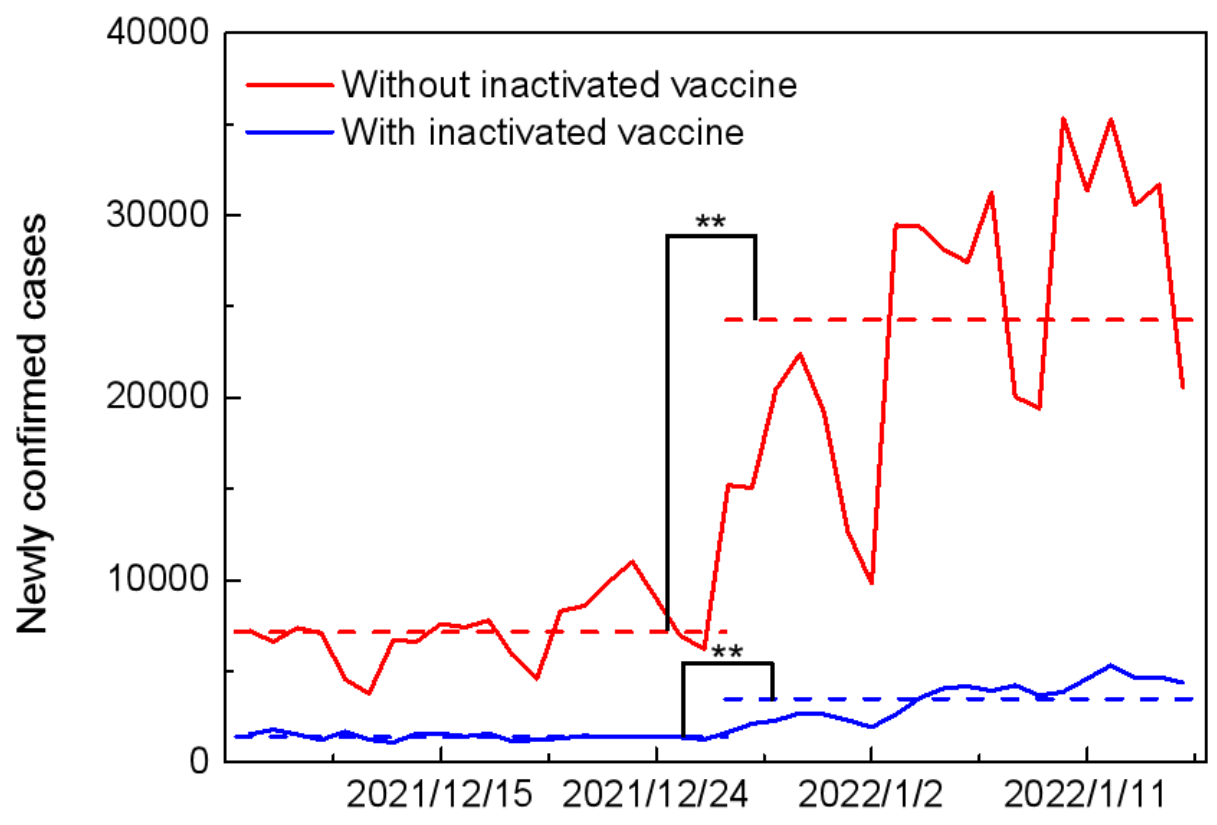

Figure 1. Comparison of time series of newly confirmed cases in predominantly inactivated and non-inactivated vaccine countries. The red line shows the average number of newly confirmed cases in countries without inactivated vaccine licenses, and the blue line shows the average number of newly confirmed cases in countries using mainly inactivated vaccines.

Table 1. Vaccine types in the top 10 countries with Omicron genome sequences.

\begin{tabular}{|c|c|c|}
\hline Country & $\begin{array}{c}\text { Total \#Omicron GRA } \\
\text { (B.1.1.529+BA.*) }\end{array}$ & Vaccines Approved for Use \\
\hline United Kingdom & 191,934 & $\begin{array}{c}\text { Pfizer/BioNTech BNT162b2 } \\
\text { Moderna mRNA-1273 } \\
\text { Janssen (Johnson\& Johnson). Ad26.COV2.S } \\
\text { Oxford/AstraZeneca AZD1222 }\end{array}$ \\
\hline USA & 131,783 & $\begin{array}{c}\text { Pfizer/BioNTech BNT162b2 } \\
\text { Moderna mRNA-1273 } \\
\text { Janssen (Johnson\& Johnson). Ad26.COV2.S }\end{array}$ \\
\hline Denmark & 28,464 & $\begin{array}{c}\text { Moderna mRNA-1273 } \\
\text { Pfizer/BioNTech BNT162b2 } \\
\text { Novavax NVX-COV2373 } \\
\text { Janssen (Johnson \&Johnson) Ad26.COV2.S }\end{array}$ \\
\hline Germany & 10,455 & $\begin{array}{c}\text { Moderna mRNA-1273 } \\
\text { Pfizer/BioNTech BNT162b2 } \\
\text { Novavax NVX-COV2373 } \\
\text { Janssen (Johnson \&Johnson) Ad26.COV2.S } \\
\text { Oxford/AstraZeneca AZD1222 }\end{array}$ \\
\hline France & 9,073 & $\begin{array}{c}\text { Moderna mRNA-1273 } \\
\text { Pfizer/BioNTech BNT162b2 } \\
\text { Novavax NVX-COV2373 } \\
\text { Janssen (Johnson \&Johnson) Ad26.COV2.S } \\
\text { Oxford/AstraZeneca AZD1222 } \\
\end{array}$ \\
\hline Canada & 6,406 & $\begin{array}{c}\text { Moderna mRNA-1273 } \\
\text { Pfizer/BioNTech BNT162b2 } \\
\text { Janssen (Johnson \&Johnson) Ad26.COV2.S } \\
\text { Oxford/AstraZeneca AZD1222 }\end{array}$ \\
\hline
\end{tabular}




\begin{tabular}{|c|c|c|}
\hline & & Serum Institute of India Covishield (Oxford/AstraZenecaformulation) \\
\hline Australia & 5,720 & $\begin{array}{c}\text { Moderna mRNA-1273 } \\
\text { Pfizer/BioNTech BNT162b2 } \\
\text { Janssen (Johnson \&Johnson) Ad26.COV2.S } \\
\text { Oxford/AstraZeneca AZD1222 }\end{array}$ \\
\hline Switzerland & 5,409 & $\begin{array}{c}\text { Moderna mRNA-1273 } \\
\text { Pfizer/BioNTech BNT162b2 } \\
\text { Janssen (Johnson \&Johnson) Ad26.COV2.S }\end{array}$ \\
\hline Israel & 4,860 & $\begin{array}{c}\text { Moderna mRNA-1273 } \\
\text { Pfizer/BioNTech BNT162b2 }\end{array}$ \\
\hline Italy & 3,733 & $\begin{array}{c}\text { Moderna mRNA-1273 } \\
\text { Pfizer/BioNTech BNT162b2 } \\
\text { Novavax NVX-COV2373 } \\
\text { Janssen (Johnson \&Johnson) Ad26.COV2.S } \\
\text { Oxford/AstraZeneca AZD1222 }\end{array}$ \\
\hline
\end{tabular}

\subsection{Global epidemic and vaccine distribution characteristics}

Figure 2 shows the global spatial distribution of inactivated vaccination rates and newly confirmed cases. Countries with higher rates of inactivated vaccination have fewer new confirmed cases. There are no major outbreaks and in countries such as China, Chad, Algeria, Niger, Venezuela, and Paraguay where inactivated vaccines are the majority. They effectively withstand this serious disaster. However, the United States, Canada, Australia and most European countries are in the midst of their worst outbreaks in two years, and none of these countries has licensed an inactivated vaccine. After being swept by Omicron, those countries had about 50 times more newly confirmed cases in 20 days than other low-risk countries. This further demonstrates the broad spectrum advantage of inactivated vaccines against highly mutated viral variants.

In Japan, where COVID-19 has been strictly controlled, the number of daily new cases has exceeded 80,000 in this omicron outbreak, which is 3.5 times the previous highest number of daily new cases in Japan. Current licensed vaccines in Japan mainly consist of Takeda TAK-919 (Moderna formulation), RNA vaccines from Pfizer/BioNTech Comirnaty and Replicating Viral Vector vaccines from Oxford/AstraZeneca VaxzevriaNon, also not including inactivated virus vaccines. India also experienced a severe third outbreak, with the number of new cases reaching as high as 340,000 in a single day. India, with the world's second largest population, has a high residential density, health inequity, growing economic and social disparities, pose challenges to the response to the epidemic. As of the time of the omicron outbreak, only $41.3 \%$ of people with a complete vaccination. Of the 10 vaccines licensed, only 1 is inactivated (Bharat Biotech, Covaxin). 
a

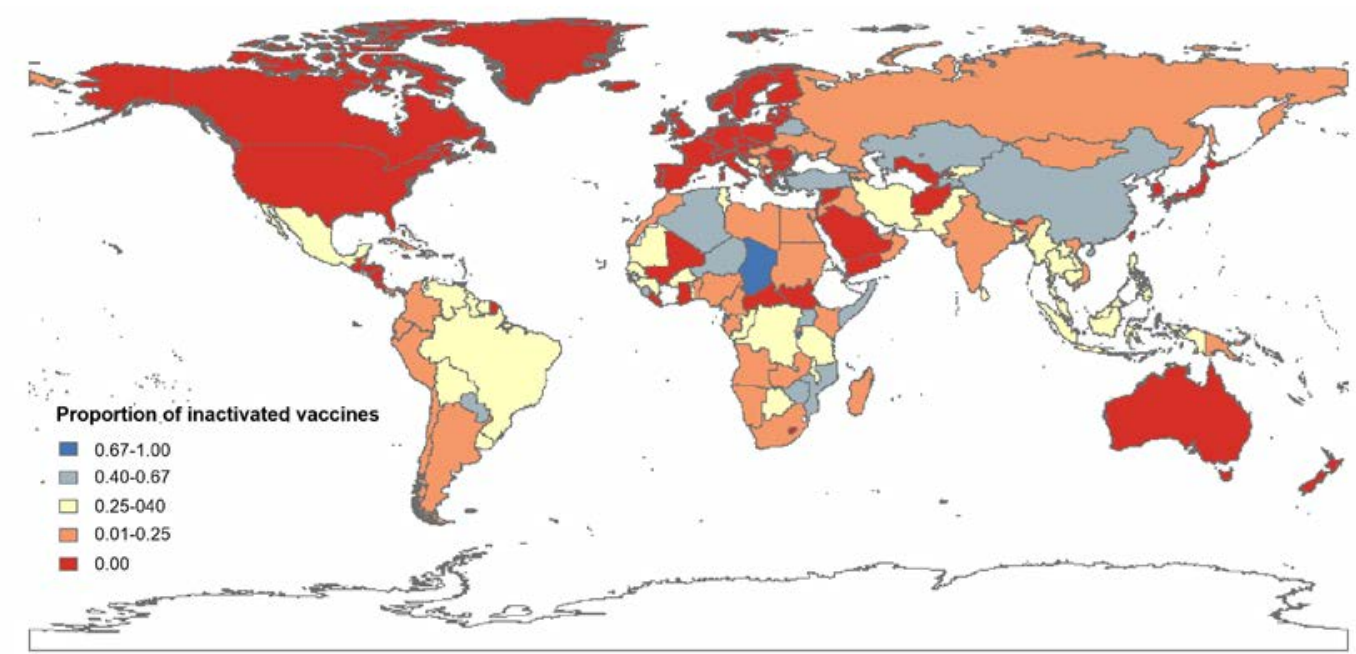

b

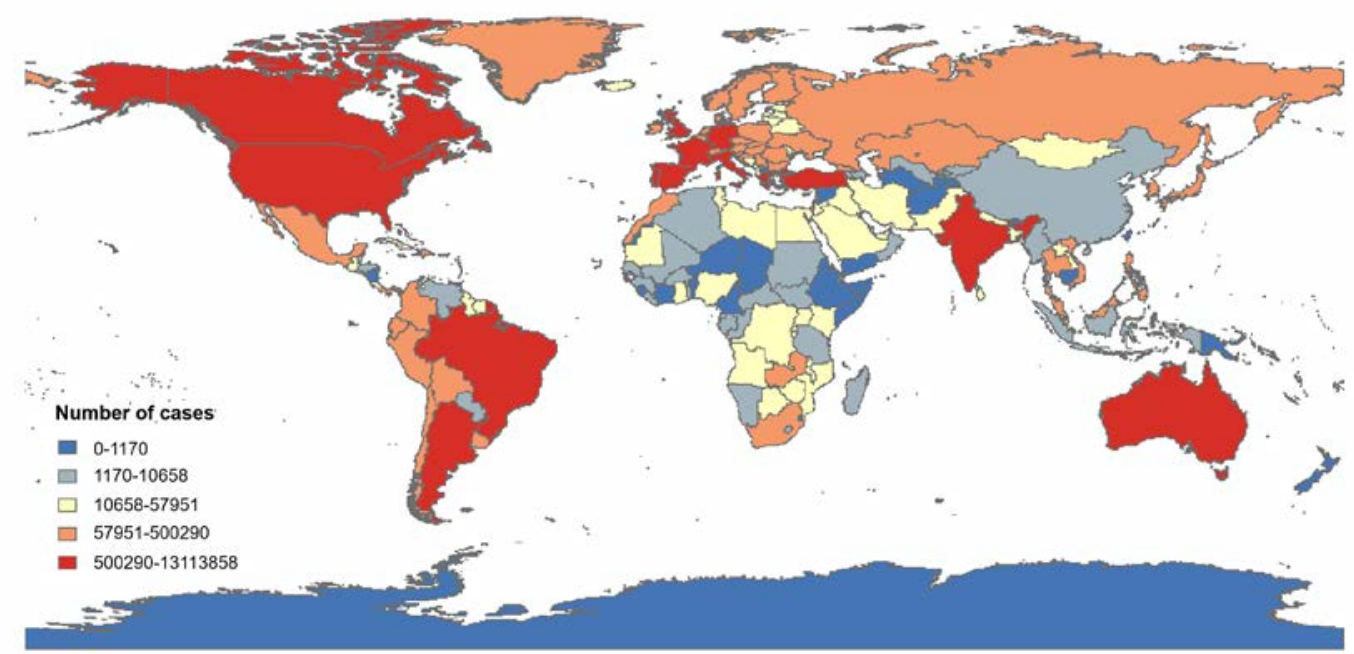

Figure 2. Global spatial distribution of vaccination and confirmed cases. a, Spatial distribution of inactivated vaccine proportions; $b$, Spatial distribution of newly confirmed cases.

\subsection{Characteristics of the Epidemic in China}

Figure 3 shows the spread of Omicron in China. Varying from other countries, the number of infections in China has remained low and stable during the Omicron spreading, without significant difference from the previous spread of delta variant in China. In the two months since the Omicron variant was defined as VOC, local Omicron cases in China (mainly in areas where inactivated vaccines have been used) accounted for 31.6\% of the total new cases. The cumulative confirmed Omicron cases were 361 and 463 in Tianjin city and Henan province, where the Omicron variant caused the most local infections, accounted for $74.4 \%$ and $45.9 \%$ of the local COVID-19 cases (Figure 2). While the average proportion of Omicron sequences in the global hardest-hit countries is about $72.7 \%$. The Omicron GRA (B.1.1.529+BA. ${ }^{*}$ ) percentages is $98.9 \%$ and $99.2 \%$ in the US and the UK, respectively.

The Chinese government has effectively responded to the Omicron outbreak by adopting timely and effective non-pharmaceutical interventions.. On 10 January, 2022, the Chinese government issued the travel restriction to and from Tianjin, to contain the 21 
new Omicron infections recorded there on 9 January. In Anyang, a city of 5.5 million people, all bus services had been suspended. In addition, citizens need to work from home and actively participate in multiple rounds of nucleic acid tests. Effective pharmaceutical interventions in China, including the usage of inactivated vaccines, have also played a significant role. Australia also had similarly implemented strict lockdowns and tight border controls earlier in the pandemic. However, the Omicron has accelerated the unraveling of the country's aggressive containment efforts [18]. The fact is that inactivated vaccines are not licensed in Australia. This may be further evidence that the broad spectrum of the inactivated vaccine has kept Omicron from causing large-scale outbreaks in China.

a

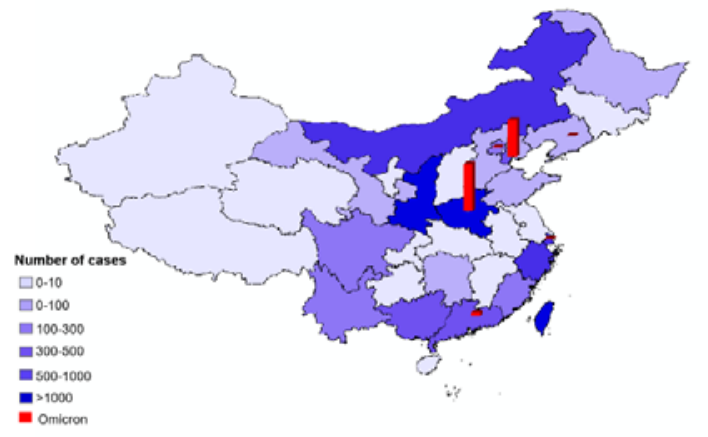

b

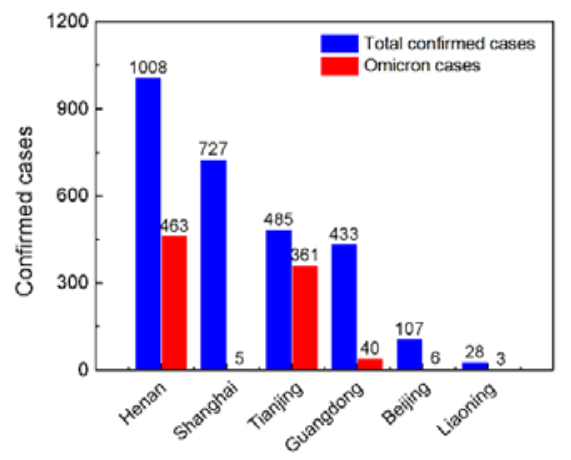

Figure 3. Spatial distribution of the epidemic in China. a, Spatial distribution of newly confirmed cases in China since the first Omicron case. The red bar represents Omicron cases and the blue grading represents the total number of new cases. $b$, Distribution of newly confirmed cases in provinces with Omicron cases in China. Red represents Omicron cases and blue represents the total number of new cases.

\subsection{Simulation of vaccine protection}

Figure 4 presents the simulation of the prevention effect of inactivated vaccines on epidemic development. The simulation result shows that inactivated vaccines avoided an outbreak of about 1.6 million cases in Hangzhou, China. An epidemic caused by the omicron variant occurred in Hangzhou, China, in January 2022, which lasted nine days and the cumulative number of confirmed cases was 114. By introducing the parameters of the government's control measures, the simulation result of the cumulative confirmed cases was 116 , with an accuracy rate of $98.2 \%$. Without strict government controls, the epidemic in Hangzhou would have subsided around February 23, 2022, with the cumulative number of infections reaching around 90,000. We further simulated the Hangzhou outbreak using transmission patterns in non-inactivated vaccine countries without considering control measures. The simulation result shows that the daily new cases are expected to peak at around 250,000, and the cumulative infections will exceed 1.7 million on February 23, 2022, which is nearly 20 times different from the simulation results in countries using inactivated vaccines.

In addition to immune escape, mutations of omicron variant significantly increase the binding free energy (BFE) between the S RBD and the ACE2 changes to enhance its evolutionary advantages at the RBD $[19,20]$. The accumulated BFE change is $2.60 \mathrm{kcal} / \mathrm{mol}$, suggesting over 10 times more contagious than the original virus or about 2.8 times as infectious as the Delta variant (BFE change: $1.57 \mathrm{kcal} / \mathrm{mol}$ ) [11]. However, when infection rate $\beta$ is set at 2.8 times the Delta strain, the simulation is consistent with the omicron 
outbreak in non-inactivated vaccine countries, but could not simulate the epidemic trend in China, indicating that the protection of inactivated vaccine in China was effective.

a

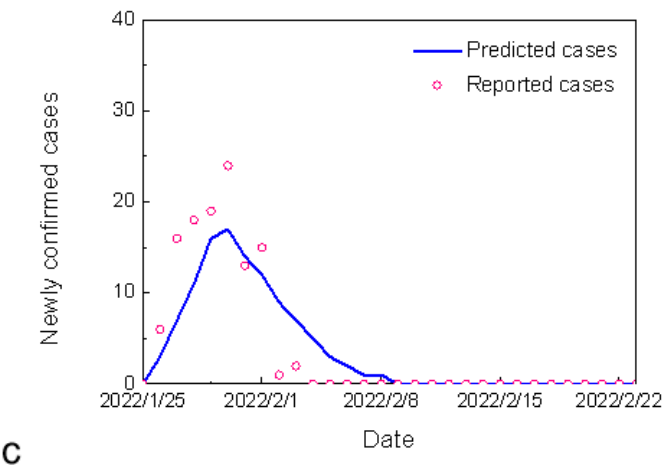

C

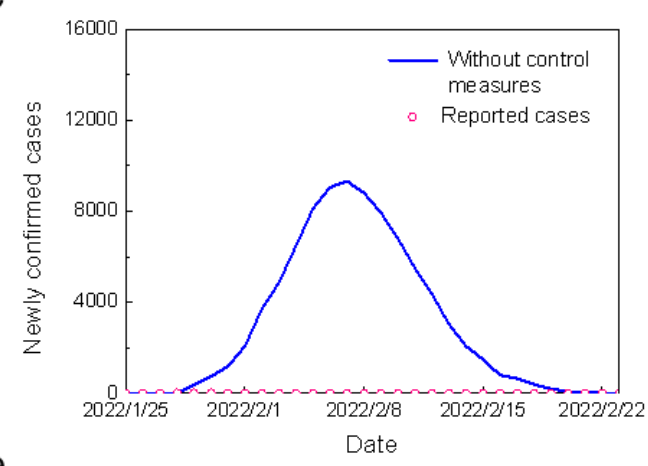

e

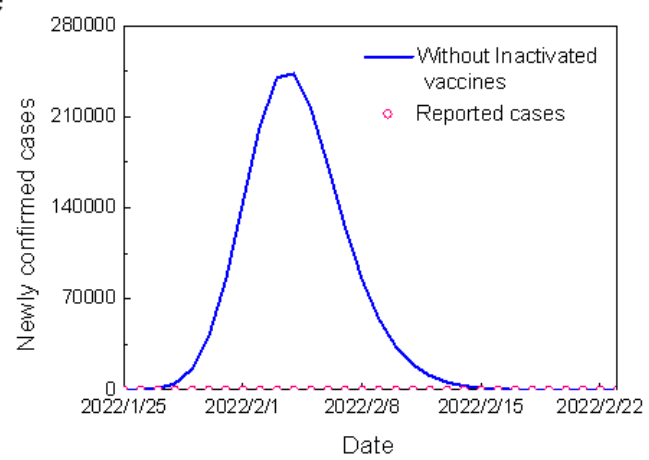

b

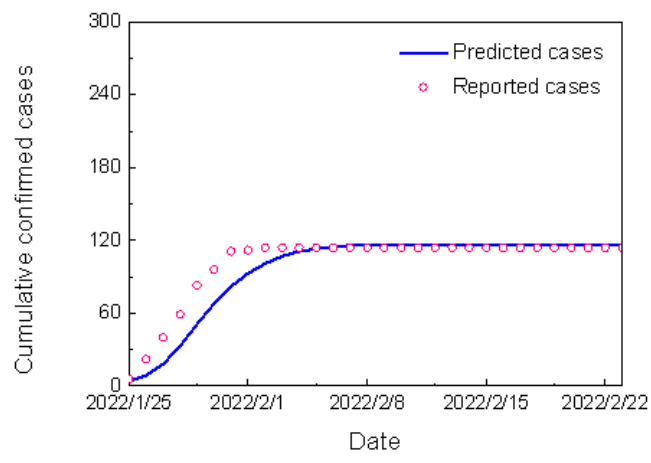

d
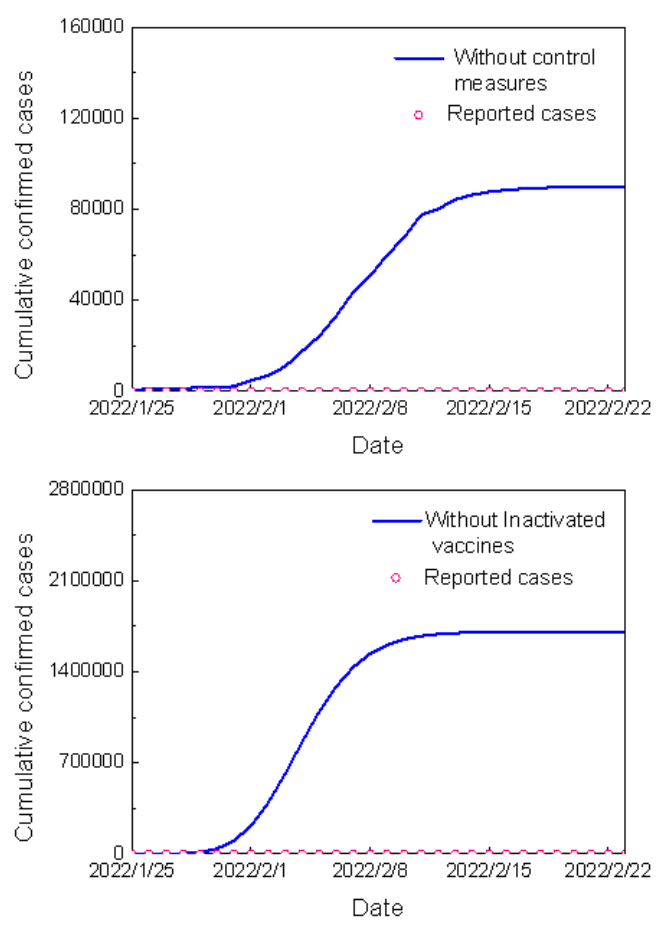

Figure 4. Simulation of the epidemic in Hangzhou, China. a, Simulation prediction of newly confirmed cases in Hangzhou. $b$, Simulation prediction of cumulative confirmed cases in Hangzhou. c, Simulation of newly confirmed cases in Hangzhou without control measures. d, Simulation of cumulative confirmed cases in Hangzhou without control measures. e, Simulation of newly confirmed cases in Hangzhou without Inactivated vaccines. f, Simulation of cumulative confirmed cases in Hangzhou without Inactivated vaccines.

\section{Discussion}

Viruses keep evolving to become more infectious and overcome a host's immune responses [21]. Evolution of variants could be possibilities in the nonhuman species or a remote population with huge evolutionary pressure, and low vaccine coverage or inequity in vaccination may provide a fertile environment in the evolution [22]. Mass infection, vaccination, and government interventions around the world are changing SARS-CoV-2's evolutionary landscape, increasing its chances of achieving a major evolutionary leap 
$[21,23]$. The future evolution of SARS-CoV-2 will determine the outcome of this global crisis. It could either turn into another common cold through universal immunization, or a more threatening global catastrophe. Herd immunity is likely to sacrifice many lives. Therefore, effective medical treatment and non-pharmaceutical interventions are the only way to defeat the pandemic [24,25].

Current vaccines are not a complete and permanent barrier. We need to develop a safe vaccine that can resist virus evolution and mutation over the long term. The success of the inactivated virus vaccine in China provides support for the development of future vaccines. Strategy of inactivated vaccine contrasts with many other COVID-19 vaccine development efforts involving their vaccine candidates' RNA. A theoretical advantage of inactivated vaccines is that they contain additional viral proteins, including nucleoprotein, which could broaden protection beyond anti-spike protein responses and reduce the escape of variants from vaccine immunity. Recent studies have shown that two doses of the inactivated vaccine, followed by a third dose of an mRNA vaccine, markedly increased the levels of specific antibodies and B and T cell responses and may thus increase protection against emerging SARS-CoV-2 variants including Omicron [26]. Rapid, simultaneous, and widespread deployment of additional vaccine doses is now needed to curtail the emergence and spread of highly mutated SARS-CoV-2 variants. Meanwhile, rigorous non-pharmaceutical interventions remain the most timely and effective responses to tsunami-like outbreaks worldwide.

Author Contributions: Conceptualization, Jianping Huang; Data curation, Shuoyuan Gao and Xiaodong Song; methodology, Li Zhang; visualization, Xinbo Lian; writing-original draft preparation, Xinbo Lian; writing - review and editing, Xinbo Lian, Jianping Huang, Yingjie Zhao, Danfeng Wang and Rui Wang. All authors have read and agreed to the published version of the manuscript.

Funding: This research was funded by the National Science Foundation of China, grant number 41521004. This research was funded by the the Gansu Provincial Special Fund Project for Guiding Scientific and Technological Innovation and Development, grant number 2019ZX-06.

Data Availability Statement: The data used in this article is publicly accessible.We selected the data of vaccines approved for use in each country as the theoretical basis for the global distribution of inactivated vaccines, as there are no comprehensive data on global vaccination rates for each type of vaccine. The COVID-19 dataset is collected from the COVID-19 Data Repository by the Center for Systems Science and Engineering (CSSE) at Johns Hopkins University which illustrates the number of confirmed COVID-19 cases, deaths, and recovered cases for all affected countries in real-time (https://github.com/CSSEGISandData/COVID-19). The number and type of vaccines approved for use in each country are derived from: https://covid19.trackvaccines.org/trials-vaccines-by-country/. As of 10 February, 2022, 146 countries have shared 1,116,011 Omicron gene sequences from sample collection for public access via GISAID EpiCoV. Gene sequence data for each country were obtained from https://www.gisaid.org/hcov19-variants/.

Acknowledgments: The authors acknowledge the National Science Foundation of China and the Gansu Provincial Special Fund Project for Guiding Scientific and Technological Innovation and Development for funding the research. The authors acknowledge the Center for Systems Science and Engineering (CSSE) at Johns Hopkins University for providing the COVID-19 data.

Conflicts of Interest: The authors declare no conflict of interest. The authors declare that they have no known competing financial interests or personal relationships that could have appeared to influence the work reported in this paper.

\section{References}

1. Maslo C, Friedland R, Toubkin M, Laubscher A, Akaloo T, Kama B. Characteristics and Outcomes of Hospitalized Patients in South Africa During the COVID-19 Omicron Wave Compared With Previous Waves. Jama 2021.

2. Burki TK. Omicron variant and booster COVID-19 vaccines. The Lancet Respiratory Medicine 2021.

3. Kumar S, Thambiraja TS, Karuppanan K, Subramaniam G. Omicron and Delta variant of SARS-CoV-2: a comparative computational study of spike protein. Journal of medical virology 2021.

4. Rogliani P, Chetta A, Cazzola M, Calzetta L. SARS-CoV-2 Neutralizing Antibodies: A Network Meta-Analysis across Vaccines. Vaccines 2021, 9, 227. 
5. Boschiero N. COVID-19 Vaccines as Global Common Goods: An Integrated Approach of Ethical, Economic Policy and Intellectual Property Management. Global Jurist 2021.

6. Fakhroo A, AlKhatib H A, Al Thani A A, et al. Reinfections in COVID-19 Patients: Impact of Virus Genetic Variability and Host Immunity. Vaccines 2021, 9, 1168.

7. Naranbhai V, Pernat CA, Gavralidis A, et al. Immunogenicity and reactogenicity of SARS-CoV-2 vaccines in patients with cancer: the CANVAX Cohort Study. Journal of Clinical Oncology 2022, 40, $12-23$.

8. Garcia-Beltran WF, Denis KJS, Hoelzemer A, et al. mRNA-based COVID-19 vaccine boosters induce neutralizing immunity against SARS-CoV-2 Omicron variant. Cell 2021.

9. Huang J., L. Zhang, X. Liu, et al. Global prediction system for COVID-19 pandemic. Sci. Bull 2020, 65, 1884-1887.

10. Peng L., W. Yang, D. Zhang, et al. Epidemic analysis of COVID-19 in China by dynamical modeling. medRxiv 2020, 1-18.

11. Chen J, Wang R, Gilby N B, et al. Omicron variant (B. 1.1. 529): Infectivity, vaccine breakthrough, and antibody resistance. Journal of chemical information and modeling 2022.

12. Garcia-Beltran W F, Denis K J S, Hoelzemer A, et al. mRNA-based COVID-19 vaccine boosters induce neutralizing immunity against SARS-CoV-2 Omicron variant. Cell 2022.

13. Cele S, Jackson L, Khoury D S, et al. Omicron extensively but incompletely escapes Pfizer BNT162b2 neutralization. Nature 2021, $1-5$.

14. Mok CKP, Cohen CA, Cheng SM, et al. Comparison of the immunogenicity of BNT162b2 and CoronaVac COVID-19 vaccines in Hong Kong. Respirology 2021.

15. Nguyen TH, Rowntree LC, Petersen J, et al. CD8+ T cells specific for an immunodominant SARS-CoV-2 nucleocapsid epitope display high naive precursor frequency and TCR promiscuity. Immunity 2021, 54, 1066-82. e5.

16. Nikiforow S, Bottomly K, Miller G, Münz C. Cytolytic CD4+-T-cell clones reactive to EBNA1 inhibit Epstein-Barr virus-induced B-cell proliferation. Journal of virology 2003, 77, 12088-104.

17. Peng Y, Mentzer AJ, Liu G, et al. Broad and strong memory CD4 ${ }^{+}$and CD8 ${ }^{+} \mathrm{T}$ cells induced by SARS-CoV-2 in UK convalescent individuals following COVID-19. Nature immunology 2020, 21, 1336-45.

18. Taylor L. Covid-19: Omicron drives weekly record high in global infections. BMJ 2022, 376, o66.

19. Li C, Tian X, Jia X, et al. The impact of receptor-binding domain natural mutations on antibody recognition of SARS-CoV-2. Signal transduction and targeted therapy 2021, 6, 1-3.

20. Wang R, Chen J, Gao K, et al. Vaccine-escape and fast-growing mutations in the United Kingdom, the United States, Singapore, Spain, India, and other COVID-19-devastated countries. Genomics 2021, 113, 2158-2170.

21. Callaway E. Beyond Omicron: what's next for COVID's viral evolution. Nature 2021, 600, 204-7.

22. Thakur V, Kanta Ratho R. Omicron (B. 1.1. 529): A new SARS-CoV-2 Variant of Concern mounting worldwide fear. Journal of medical virology 2021.

23. Zhao Y, Huang J, Zhang L, Chen S, Gao J, Jiao H. The global transmission of new coronavirus variants. Environmental Research 2021, 112240.

24. Huang J, Liu X, Zhang L, et al. The oscillation-outbreaks characteristic of the COVID-19 pandemic. National Science Review 2021.

25. Lian X, Huang J, Huang R, Liu C, Wang L, Zhang T. Impact of city lockdown on the air quality of COVID-19-hit of Wuhan city. Science of the Total Environment 2020, 742, 140556.

26. Zuo F, Abolhassani H, Du L, et al. Heterologous immunization with inactivated vaccine followed by mRNA booster elicits strong humoral and cellular immune responses against the SARS-CoV-2 Omicron variant. medRxiv 2022. 